\title{
Perceived Stress and Coping among Rural Adolescent Girls in India
}

\author{
G. Ragesh ${ }^{1}$, C. Sabitha ${ }^{2}$, Dr. A. Anithakumari ${ }^{3}$, Dr. Ameer Hamza ${ }^{4}$
}

\section{ABSTRACT:}

A cross sectional study was conducted among 120 adolescent girls from rural area (of Calicut, Kerala), India. Findings showed lesser level of stress but adopted maladaptive coping strategies. Importance of mental health programme in schools and colleges targeting adolescent girls was discussed.

Keywords: stress, coping, rural adolescent girls.

Happy and confident adolescents are most likely to grow into happy and confident adults, who in turn contribute to the health and well-being of nations (1). Adolescent stresses are from within and from the various social spheres in which the adolescent operates (2). Stressful experiences and efforts to cope with stress are central to understanding psychological distress and psychopathology during adolescence (1). Mental health problems among adolescents carry high social and economic costs, as they often develop into more disabling conditions later in life (3). Studies on stress and coping of adolescents girls especially from rural back ground is very minial in Indian setting. This cross sectional study was aimed to understand the perceived stress and coping of adolescent girls from rural background in India.

\section{METHODOLOGY}

The study was conducted in a rural area, i.e. Balussery block panchayath which is about 30 kilometers away from the city area of Kozhikode (Calicut) district of Kerala state, India. A camp was organized to enhance the life skills of girls with in an age gap of 12 to 19 years. All parents of the respondents were informed about the camp and study and received assent from the -

${ }^{1} \mathrm{Ph} . \mathrm{D}$ Scholar, Department of Psychiatric Social Work, National Institute of Mental Health and Neuro Sciences (NIMHANS),Bengaluru, Karnataka State, India. PIN-560029)

${ }^{2}$ School Counselor, ICDS, Balussery, Calicut, Kerala.)

${ }^{3}$ Assistant Professor, Departmet of Psychiatry, Govt. Medical College, Calicut, Kerala.)

${ }^{4}$ Associate Professor, Department of Psychiatric Social Work, National Institute of Mental Health and Neuro Sciences (NIMHANS), Bengaluru, Karnataka State, India.) 


\section{Perceived Stress and Coping among Rural Adolescent Girls in India}

-adolescents and consent from the parents. All the girls who were diagnosed to have any kind of mental and physical health problems or disability were excluded from the study. On an average 150 girls attended the camp. All were asked to complete the questionnaire before conducting the training programme. Considered 120 data for analysis as others were incomplete. Sociodemographic profile which was prepared by researchers and Perceived Stress Scale (4) and Brief Cope Scale (5) were used to collect the data.

\section{RESULTS}

Sociodemographic details show that $29.2 \%$ of the respondents belong to the age group of $12-14$ years, $36.7 \%$ belong to the age group of $14-16$ years, $27.5 \%$ belong to the age group of $16-18$ and the rest of them above 18 years of age. The majority (66\%) belong to the Hindu religion, from nuclear family $(83.3 \%)$ and from the family of above the poverty line $(66.7 \%)$. Both the parents are present in the family for the majority (96.7\%). Most of (98.3\%) them have at least one sibling. Birth order wise, $49 \%$ of them are the first , $42 \%$ of them are second and rest are in the third position of their siblings. With regard to the substance use among fathers; $16.7 \%$ fathers abuse nicotine, $8.3 \%$ abuse alcohol and 1.7\% abuse both alcohol and nicotine. Few $(8.3 \%)$ of their family members are with chronic illness. Only one respondent with family history of suicide and mental illness. No respondent reported substance use.

With regard to their perceived stress and coping; the average score of perceived stress is 16.42 and the coping strategies like self distraction, denial, ventilation, positive reframing, self blaming, accepting and religious way of coping have significant correlation with perceived stress (table 1).

\section{DISCUSSION}

There is a dearth of literature on the coping and perceived stress among adolescent girls from rural background in India. The current study has made an attempt to understand the perceived stress and coping among adolescent girls from rural background in Indian settings.

The major finding in this study is that the respondents did not report any major stress at present compared to the findings in another study which was conducted in India that the school students in India have a high stress level (6). This may be because of the most of the respondents are from a rural back ground, family of above poverty line, both the parents are alive and living together, 


\section{Perceived Stress and Coping among Rural Adolescent Girls in India}

low rate of substance abuse in family members and the competition in academics and related stress are lesser in rural areas compared to urban areas.

The study found that the respondents adopt maladaptive coping mechanisms and most of the maladaptive coping strategies have strong correlation with stress. This findings go along with other studies (7-9) that the girls adopt more maladaptive coping mechanisms. A study conducted in india also reveals that the adolescent girls from rural areas adopt unhealthy coping styles (2). As adolescence is the time of faster biological developments and the maladaptive coping may lead to development of any from of psychopathology. This indicates the importance of mental health programme among adolescent girls. School/college mental health programme is essential to enhance the mental health status of adolescents. And it also indicates the urgent need of larger studies in rural settings.

\section{CONCLUSION}

The current study shows that the stress among adolescent girls in rural area is less but they adopt maladaptive coping strategies. There is greater opportunity for mental health promotion and preventive services among adolescents girls in schools and colleges in India.

\section{REFERENCES}

Rao M. Book Review. Promoting Children's Emotional Well-Being Ann Buchanan and Barbara Hudson. Journal of Public Health. 2001;23(2):168-9.

Samata Srivastava DJ, Srivastava OP. STRESS AND COPING STYLE OF URBAN AND RURAL ADOLESCENTS.

Kumar V, Talwar R. Determinants of psychological stress and suicidal behavior in Indian adolescents: a literature review. Journal of Indian Association for Child \& Adolescent Mental Health. 2014;10(1).

Cohen S, Kamarck T, Mermelstein R. A global measure of perceived stress. Journal of health and social behavior. 1983:385-96.

Carver CS. You want to measure coping but your protocol'too long: Consider the brief cope. International journal of behavioral medicine. 1997;4(1):92-100.

Arun P, Chavan B. Stress and suicidal ideas in adolescent students in Chandigarh. Indian journal of medical sciences. 2009;63(7):281. 
Hampel P, Petermann F. Age and gender effects on coping in children and adolescents. Journal of Youth and Adolescence. 2005;34(2):73-83.

Corbett EL, Marston B, Churchyard GJ, De Cock KM. Tuberculosis in sub-Saharan Africa: opportunities, challenges, and change in the era of antiretroviral treatment. The Lancet. 2006;367(9514):926-37.

Dubat K, Punia S, Goyal R. A study of life stress and coping styles among adolescent girls. J Soc Sci. 2007;14(2):191-4.

Table 1

Correlation

\begin{tabular}{|c|l|l|}
\hline SI no. & Coping scales domains & Stress score (16.42) \\
\hline 1. & Self distraction & $.344^{* *}$ \\
\hline 2. & Active coping & .101 \\
\hline 3. & Denial & $.264^{* *}$ \\
\hline 4. & Substance use & -.017 \\
\hline 5. & Emotional support & .048 \\
\hline 6. & Behavioural disengagement & .140 \\
\hline 7. & Ventilation & $.244^{* *}$ \\
\hline 8. & Instrumental support & .014 \\
\hline 9. & Positive reframing & $.194^{*}$ \\
\hline 10. & Planning & .147 \\
\hline 11. & Self blaming & $.269^{* *}$ \\
\hline 12. & Acceptance & $.251^{* *}$ \\
\hline 13. & Humor & .136 \\
\hline 14. & Religion & $.180^{*}$ \\
\hline
\end{tabular}

** Correlation is significant at the .01 level (2-tailed).

* Correlation is significant at the .05 level (2-tailed). 